\title{
COMPORTAMIENTO EXPERIMENTAL DE CONEXIONES DE MARCOS DE ACERO ANTE DEMANDAS SÍSMICAS
}

\author{
Edgar Tapia Hernández, ${ }^{(1)}$, Alejandro Santiago Flores ${ }^{(2)}$, Héctor Guerrero Bobadilla ${ }^{(3)}$, \\ y Marcos M. Chávez Cano ${ }^{(3)}$
}

\begin{abstract}
RESUMEN
Se discute el comportamiento inelástico cíclico de seis especímenes de conexiones viga-columna ensayados experimentalmente en escala natural. Las configuraciones seleccionadas son ampliamente empleadas en estructuras de acero con marcos momento resistentes en la práctica mexicana. De acuerdo a los resultados, las conexiones ensayadas bajo grandes deformaciones inelásticas desarrollaron una concentración de daño inesperada que no es lo suficientemente dúctil para una estructura de acero de alta ductilidad. Se enfatiza el efecto de algunos parámetros de soldadura y detalles de fabricación que mejorarían la respuesta de las conexiones ante demandas sísmicas.
\end{abstract}

Palabras clave: marcos de acero; demandas sísmicas; conexiones; prueba experimental; ductilidad

\section{EXPERIMENTAL BEHAVIOR OF CONNECTIONS FOR STEEL FRAMES UNDER SEISMIC DEMANDS}

\begin{abstract}
Cyclic inelastic behavior of six beam-column connections experimentally tested through naturalscale models are discussed. Selected configurations are widely employed in moment-resisting steel frame structures by the Mexican practice. According to the results, tested moment-resisting connections under large inelastic deformations demands developed an unexpected damage concentration that is not enough ductile for a high-ductility steel structure. The effect of various welding parameters and fabrications details are emphasized in order to improve the response of the connections under seismic demands.
\end{abstract}

Keywords: steel frames; earthquake demands; connections; experimental test; ductility

Artículo recibido el 17 de enero del 2020 y aprobado para su publicación el 17 de septiembre de 2020. Se aceptarán comentarios y/o discusiones hasta cinco meses después de su publicación.

(1) Profesor - investigador, Laboratorio de Estructuras, Universidad Autónoma Metropolitana - Azcapotzalco. etapiah@azc.uam.mx

(2) Estudiante de maestría. Universidad Nacional Autónoma de México. ing.alejandro.sf@gmail.com

(3) Profesor - Investigador, Instituto de Ingeniería - Universidad Nacional Autónoma de México, hguerrerob@iingen.unam.mx; mchavezc@iingen.unam.mx

DOI: $10.18867 /$ ris. 103.562 


\section{INTRODUCCIÓN}

Por muchos años, la intención básica de los reglamentos para el diseño sísmico ha sido proveer los criterios que garanticen que los edificios tengan la capacidad de resistir demandas sísmicas intensas sin colapso, pero permitiendo daño estructural significativo. Con este propósito, se direcciona el uso de configuraciones de edificios, sistemas estructurales, materiales y detalles que sean capaces de desarrollar un comportamiento dúctil; esto es, un sistema que logre grandes deformaciones inelásticas sin una degradación significativa de resistencia ni inestabilidad (e.g. García y Tapia 2019).

En el pasado, los marcos de acero soldados se clasificaban como uno de esos sistemas dúctiles y, esencialmente, se creía que eran invulnerables al daño inducido por el sismo, puesto que se esperaba que el daño estuviera limitado a la respuesta dúctil de los elementos (trabes y columnas). Con esta confianza, se construyeron alrededor del mundo grandes estructuras industriales, comerciales y edificaciones con marcos de acero momento-resistentes como sistema principal desde de los sesentas (Tapia y Tena 2001). Sin embargo, el sismo de Northridge del 17 de enero de 1994 en la costa de Estados Unidos cambió este enfoque, puesto que se reportaron fracturas frágiles en las conexiones viga - columna de marcos de acero entre los patines de la viga y los patines de la columna (FEMA 350 2000). La falla en las conexiones de los marcos de acero se extendió incluso a estructuras en regiones que habían sido sometidas a demandas moderadas de aceleración (FEMA 355D-2000). El daño inició en las conexiones a niveles muy bajos de demanda plástica y una vez formadas, esas fracturas progresaron con diferentes caminos dependiendo de las condiciones particulares (Tremblay et al. 1995; FEMA 355F 2000). Este comportamiento imprevisto en las conexiones causó alarma entre los involucrados, pese a que los edificios cumplieron la intención básica de los reglamentos: experimentaron daños estructurales sin colapsar.

En los reglamentos (incluyendo las Normas Técnicas Complementarias para el Diseño y Construcción de Estructuras de Acero, NTC-DCEA-2020), se espera que la evolución del daño esté predominada por: i) articulaciones plásticas en las vigas, ii) deformación por cortante en la zona de panel o iii) una combinación de ellos. Así que, dada la experiencia, algunas dependencias desarrollaron una serie de recomendaciones de diseño como parte de la Agencia Federal para la Administración de Emergencia de Estados Unidos (FEMA por sus iniciales en inglés). Las publicaciones relacionadas con aspectos de conexiones incluyen, por mencionar algunas:

- Recomendaciones para la evaluación sísmica y actualización de criterios para soldaduras existentes (FEMA 351). Este documento recomienda métodos para evaluar el comportamiento probable de edificios existentes ante futuros sismos y su reparación para mejorar su respuesta.

- Recomendaciones para la evaluación post-sísmica y criterios de reparación de soldaduras de marcos de acero (FEMA 352). Provee recomendaciones para las inspecciones post-sísmicas para identificar daño en marcos de acero, con el propósito de evaluar su seguridad y criterios de rehabilitación de edificios dañados.

- Estado del arte de la soldadura e inspección (FEMA 355B). Resume el conocimiento sobre las propiedades de la soldadura comúnmente empleado en la construcción, el efecto de varias de sus propiedades y la efectividad de la metodología de inspección para caracterizar la calidad de la soldadura.

- Estado del arte sobre el comportamiento de las conexiones de acero (FEMA 355D). Este documento discute el comportamiento de distintos tipos de conexiones a momento ante grandes demandas de deformación inelástica. Incluye conexiones restringidas y parcialmente restringidas con configuraciones soldadas y atornilladas con base en estudios analíticos y pruebas experimentales.

Incluso, se publicaron documentos complementarios dirigidos a inversionistas, dueños, funcionarios y otras audiencias no técnicas que necesitan comprender el problema. Por ejemplo, el FEMA 354 discute 
aspectos sociales, económicos y políticos relacionados con el comportamiento sísmico de estructuras de acero.

El estudio del comportamiento de la conexión ha sido parte fundamental en mejorar la respuesta de las estructuras de acero; de hecho, el daño en las conexiones es uno de los más costosos: requiere eliminar acabados arquitectónicos, protección de incendios, etc. Por esta razón, muchos investigadores fueron comisionados para evaluar la respuesta de las conexiones más utilizadas en Estados Unidos con uniones soldadas, atornilladas y alternativas de conexiones que aseguraran un aceptable comportamiento sísmico (Bertero et al. 1994; SAC 1995; SAC 1997; FEMA 355C 2000). Estos esfuerzos derivaron en la publicación de un conjunto de criterios para conexiones precalificadas para marcos de ductilidad alta y ductilidad intermedia (AISC 358-16), que contiene recomendaciones de diseño, dimensiones y requerimientos de diseño para tornillos y soldadura de un conjunto de conexiones que han sido ensayadas y se ha comprobado que tienen una respuesta dúctil ante demandas sísmicas.

\section{SITUACIÓN EN MÉXICO}

En México, la investigación en conexiones de acero ha sido muy poca (e.g. Martín del Campo 2013) y debido a particularidades del mercado, el conocimiento adquirido en los documentos discutidos no se ha integrado a la práctica en la mayoría de los casos. Con este panorama, las conexiones se diseñan, analizan, fabrican y montan sin conocimiento fidedigno de su respuesta ante demandas sísmicas o, en el mejor de los casos, haciendo adaptaciones del conocimiento a las condiciones locales, que no forzosamente corresponden al análisis o prueba experimental original. Por ejemplo, se adaptan las recomendaciones de conexiones de viga I a columna I del manual AISC 358-16 a conexiones de viga I a columna de sección cajón, puesto que son muy usadas en el mercado local. Aunado a esto, el hecho que las edificaciones estructuradas con acero han representado un porcentaje muy pequeño de las estructuras con daño severo o colapso en los sismos importantes en México, ocasiona que las investigaciones analíticas y experimentales centren la atención en otros sistemas estructurales. Es posible consultar más información sobre los edificios de acero dañados en el sismo del 19 de septiembre de 1985 en Osteraas y Krawinkler (1989) y sobre los edificios dañados en los sismos de septiembre de 2017 en Tapia y García (2019).

Con este panorama, esta investigación discute la respuesta de seis conexiones viga - columna en escala natural que se caracterizan por ser configuraciones muy utilizadas en el país en zonas sísmicas. El estudio pretende i) evaluar el comportamiento de conexiones basadas en las configuraciones más utilizadas en la práctica local, ii) identificar posibles procedimientos para mejorar la respuesta de las conexiones; especialmente, en edificaciones existentes y iii) establecer la estabilidad de su incursión inelástica de las conexiones típicas.

\section{ESPECÍMENES ESTUDIADOS}

Este artículo representa la primera etapa de una investigación sobre recomendaciones para mejorar la práctica local en estructuras de acero. En esta instancia no se pretendía establecer mejoras de conexiones a las configuraciones más utilizadas en la práctica mexicana, sino que el propósito principal era establecer la vulnerabilidad de las edificaciones de acero existentes en zonas sísmicas del país. Por esta razón, se realizó una encuesta con algunos de los despachos de cálculo y fabricantes más reconocidos del país en el análisis, diseño, fabricación y montaje de estructuras de acero para establecer las conexiones más utilizadas. Es posible conocer más detalles de la encuesta en Santiago (2020). 
En el consenso, se establecieron tres configuraciones como punto de partida privilegiando conexiones de viga I a columna I: un espécimen llamado CTP1, que es una conexión a momento conectado al eje mayor de la columna I y dada la incertidumbre local, se consideraron dos soluciones de conexiones a momento al eje menor de la columna I (llamadas CTP y CTA). En cada caso se ensayaron dos especímenes con las mismas características para corroborar su respuesta.

La conexión CTP1 (figura 1a) se aproxima a la conexión precalificada "Conexión a momento soldada a patín y soldada al alma", que se discute en el capítulo 8 del AISC 358-16 (conocida como WUFW por sus siglas en inglés). En esta solución, la trabe I se une al eje mayor de la columna I, donde los patines de la trabe se unen a los patines de la columna con soldadura de penetración completa. Con este propósito, se realiza una muesca en el alma de trabe, que debe atender las recomendaciones del suplemento del AWS D1.8 (2016). También, el alma de la trabe se une con cordones de soldadura al patín de columna y, en el caso de la conexión ensayada, se incluye una placa adicional que sirve para el montaje mediante cuatro tornillos. Una vez que la trabe se suelda a la columna, los tornillos ya no trabajan y podrían retirarse, pero en la práctica se acostumbra mantenerlos. En la solución, el alma de la columna tiene unos cartabones justo en la posición de los patines de la trabe como refuerzo y continuidad en la columna, que están unidos con soldadura de penetración completa en taller.

El espécimen CPA es una conexión a momento (figura 1b), donde la trabe se une al eje menor de columna. En la configuración, los fabricantes y diseñadores intentan adaptar las recomendaciones de la conexión WUF-W (AISC 358-16) a las condiciones locales, por lo que se conoce como una conexión semiprecalificada, aunque no existe referencia experimental que lo compruebe. Los patines de la trabe se unen mediante soldadura de penetración completa corrida a una placa en forma de T que sirve también como atiesador al alma de la columna. Con el propósito de que la soldadura sea corrida, es necesario hacer una muesca en el alma de trabe. Los patines de la trabe se unen al cartabón del alma de columna mediante cordones corridos de soldadura. La configuración incluye cartabones en el lado contrario de la conexión para atiesar el alma de la columna en ambos ejes. La conexión tiene igualmente cuatro tornillos que ayudan en el montaje y que después de la aplicación de la soldadura se podrían retirar.

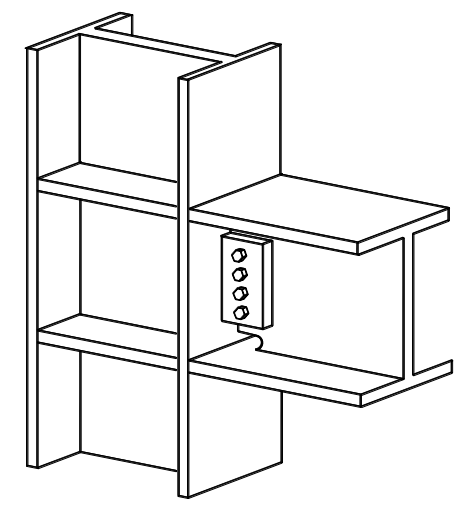

a) Espécimen CTP1

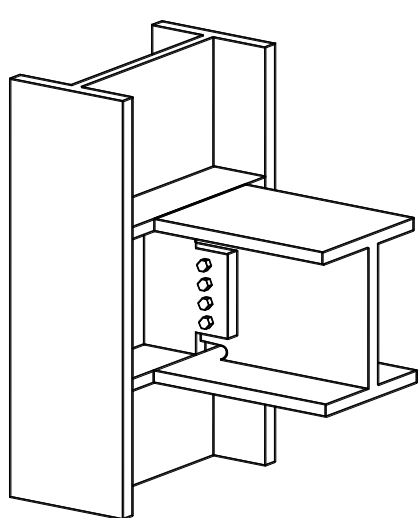

b) Espécimen CPA

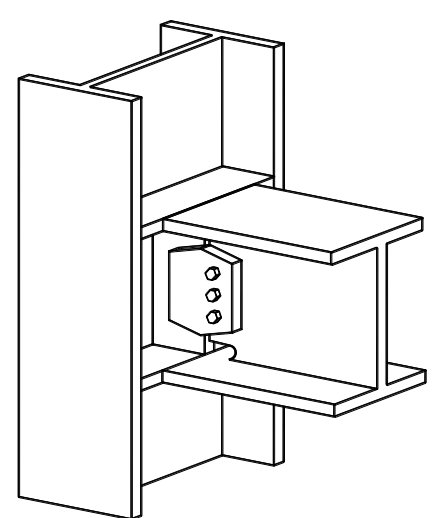

c) Espécimen CTA

Figura 1. Configuraciones ensayadas en esta investigación

Finalmente, se consideró una configuración de conexión a momento nombrada CTA (figura 1c), donde la unión se realiza mediante una placa adicional sin aristas que une el alma de la trabe a los cartabones que atiesan el alma de la columna. Los patines de la trabe se unen con soldadura de penetración completa a los cartabones del alma de la columna, por lo que el alma de la trabe debe tener muescas para que el cordón de la soldadura sea corrido entre los patines de la columna. En esta solución, el montaje se efectúa mediante tres tornillos que después de la aplicación de la soldadura se podrían retirar. 
Siguiendo el procedimiento de diseño por capacidad de las NTC-DCEA-2020 (Sec. 12.2.1), las columnas se diseñaron con la máxima capacidad que podía desarrollar la trabe considerando la formación de una articulación plástica. En el diseño se atendieron estrictamente los requisitos para marcos rígidos de ductilidad alta, usando perfiles ASTM A572 Gr. 50 (NMX-B-284), lo que incluyó los requisitos de relaciones ancho/grueso para elementos de ductilidad alta. En el proceso, el factor de sobrerresistencia del material se estableció con base en los esfuerzos reportados en los certificados de calidad del productor de los perfiles laminados, tal que para las vigas se consideró $R_{y}=1.12$. Las secciones transversales del diseño se muestran en la tabla 1.

Tabla 1. Sección transversal de los especímenes

\begin{tabular}{|c|c|c|c|c|}
\hline Espécimen & Características & Trabe & Columna & $\begin{array}{c}\text { Cantidad de } \\
\text { ensayes }\end{array}$ \\
\hline CTP1 & $\begin{array}{c}\text { Conexión al eje } \\
\text { mayor de la columna }\end{array}$ & $\begin{array}{c}\text { I 406x46.20 kg/m } \\
\text { (W 16”x31 Lb/pie) }\end{array}$ & $\begin{array}{c}\text { I 457x59.80 kg/m } \\
\text { (W 18”x40 Lb/pie) }\end{array}$ & 2 \\
\hline CPA & $\begin{array}{c}\text { Conexión al eje } \\
\text { menor de la columna }\end{array}$ & $\begin{array}{c}\text { I 305x38.70 kg/m } \\
\text { (W 12”x26 Lb/pie) }\end{array}$ & $\begin{array}{c}\text { I 356x110.40kg/m } \\
\text { (W 14”x74 Lb/pie) }\end{array}$ & 2 \\
\hline CTA & $\begin{array}{c}\text { Conexión al eje } \\
\text { menor de la columna }\end{array}$ & $\begin{array}{c}\text { I 305x38.70 kg/m } \\
\text { (W 12”x26 Lb/pie) }\end{array}$ & $\begin{array}{c}\text { I 356x110.40kg/m } \\
\text { (W 14”x74 Lb/pie) }\end{array}$ & 2 \\
\hline
\end{tabular}

\section{PROCEDIMIENTO DE ENSAYE}

Las pruebas experimentales se efectuaron en el Laboratorio de Estructuras y Materiales del Instituto de Ingeniería de la Universidad Nacional Autónoma de México (II-UNAM). Las pruebas se desarrollaron sobre la losa de reacción de planta rectangular de 6.0 x $11.0 \mathrm{~m}$ con perforaciones a cada $50 \mathrm{~cm}$ en ambas direcciones para anclar los especímenes (figura 2). En complemento, se instaló un muro de reacción modular compuesto de tres bloques de concreto reforzado de $1.5 \times 1.5 \times 1.0 \mathrm{~m}$, para sujetar un actuador dinámico con capacidad de 50 ton.

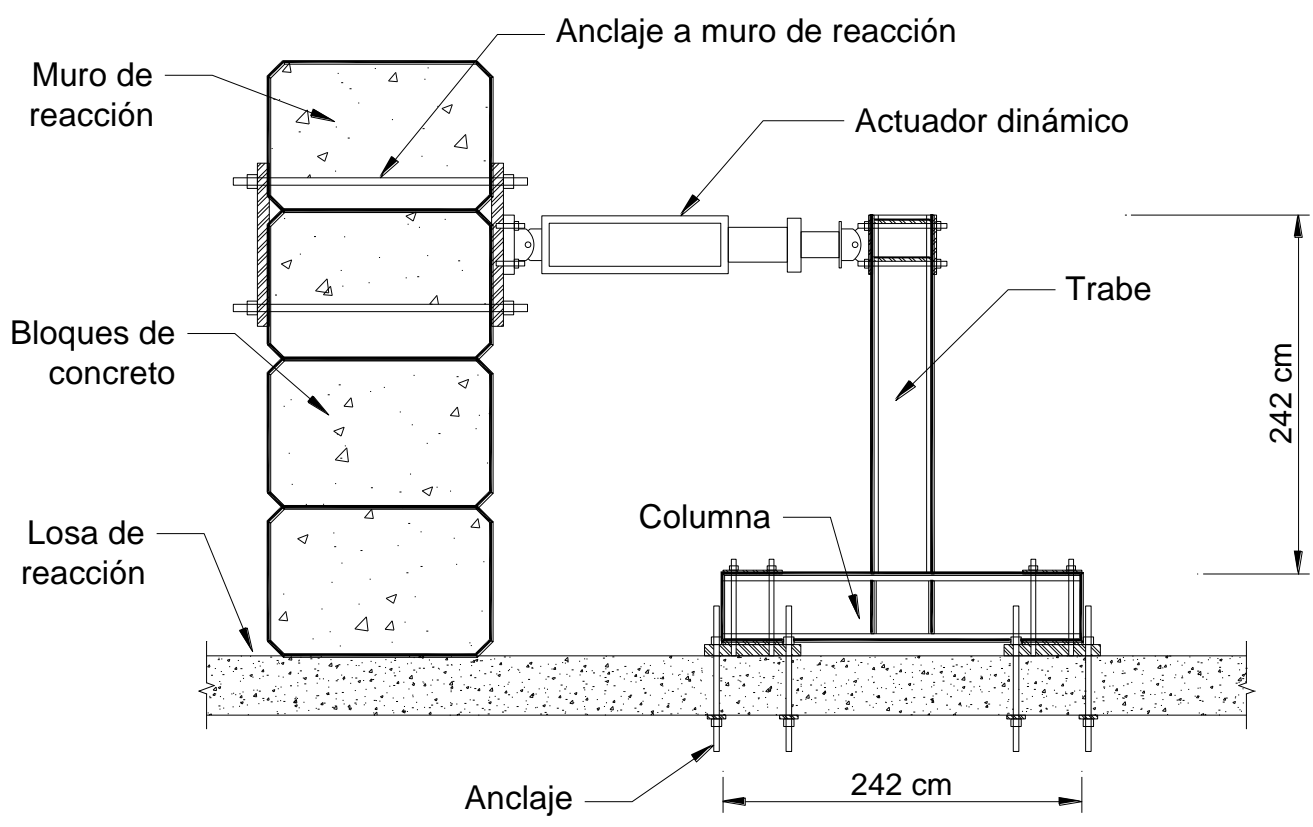

Figura 2. Configuración típica de los experimentos en el laboratorio 
La configuración de los especímenes se definió en forma de "T" invertida (figura 2), donde la columna se colocó de manera horizontal anclada en sus extremos a la losa de reacción a través de barras roscadas de alta resistencia que impiden el desplazamiento del espécimen. La viga se conectó al centro de la columna; de manera que, en el extremo libre de la viga se sujetó el actuador. La definición de las dimensiones de los especímenes consideró las condiciones del laboratorio, la facilidad en la fabricación, las condiciones del transporte y espacios del laboratorio para las maniobras y preparación. El programa incluyó el ensaye de dos especímenes idénticos para cada configuración de conexión.

\section{Protocolo de carga}

El programa de prueba y protocolo de carga siguió los criterios y requisitos especificados por el AISC 341-16 (capítulo K) para conexiones no precalificadas de acero. El protocolo de carga consiste en la aplicación de una historia de desplazamientos que simula las demandas sísmicas en la conexión. La carga comenzó con seis ciclos de $0.375 \%, 0.15 \%$ y $0.75 \%$ de distorsión (figura 3). Después se aplican cuatro ciclos de $1.0 \%$ de distorsión. Finalmente, se aplican dos ciclos de $1.5 \%, 2 \%, 3 \%, 4 \%$ y, así sucesivamente, hasta la falla de la conexión o la disminución crítica de la resistencia.

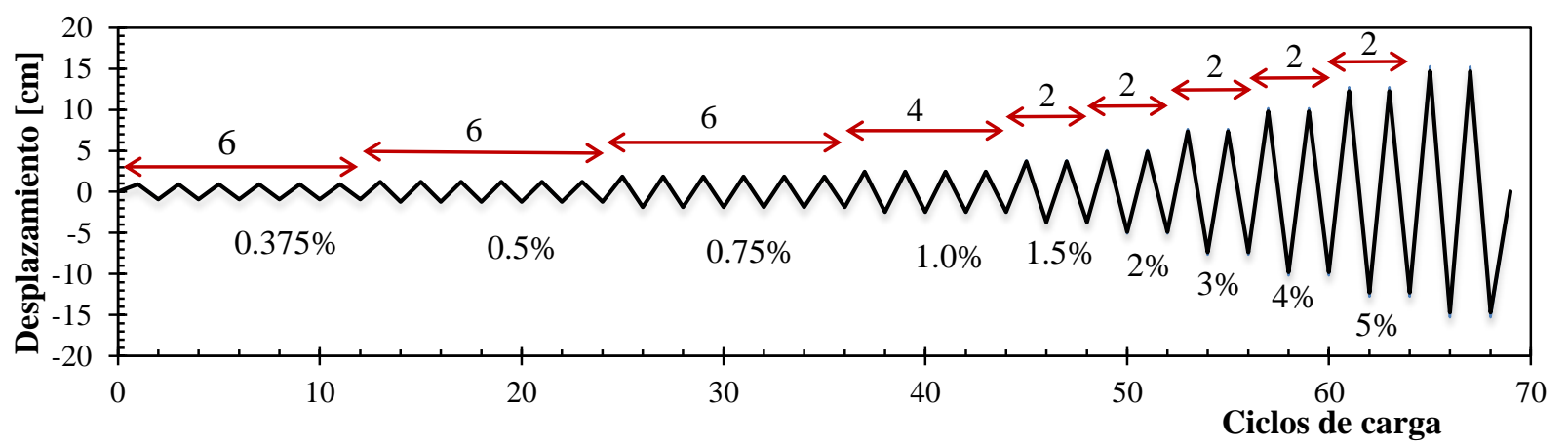

Figura 3. Protocolo de carga

\section{Instrumentación}

En los experimentos, se colocaron transductores de desplazamiento (CDP) Tokyo Sokki Kenkyujo (figura 4), en contacto con el espécimen para registrar el posible cambio de posición horizontal del espécimen. El transductor de desplazamiento CDP-1 medía el desplazamiento de la viga a la mitad de la distancia entre el eje de la columna y el eje del actuador sobre el patín de la columna. El CDP-2 se colocó a una cuarta parte de la distancia entre los ejes de la columna y el actuador. El CDP-3 se usó para medir el desplazamiento horizontal del espécimen. El CDP-4 se colocó para medir el desplazamiento horizontal de las placas de apoyo sujetas a la losa de reacción con el propósito de vigilar posibles deslizamientos. Adicionalmente, se instaló un sistema de 36 leds, que permiten medir el desplazamiento de puntos predefinidos (figura 4). Los LED-1 y LED-2 se colocaron sobre el eje del actuador en el extremo libre de la viga del espécimen para comprobar que los desplazamientos del actuador correspondan con el protocolo de carga introducido. Los LED-3 y LED-4 se colocaron de manera paralela a los CDP-1 y CDP-2 en el alma de la viga como respaldo. Los leds restantes se colocaron distribuidos en la zona de la conexión donde se espera la posible respuesta inelástica, en función de la configuración de la conexión. 


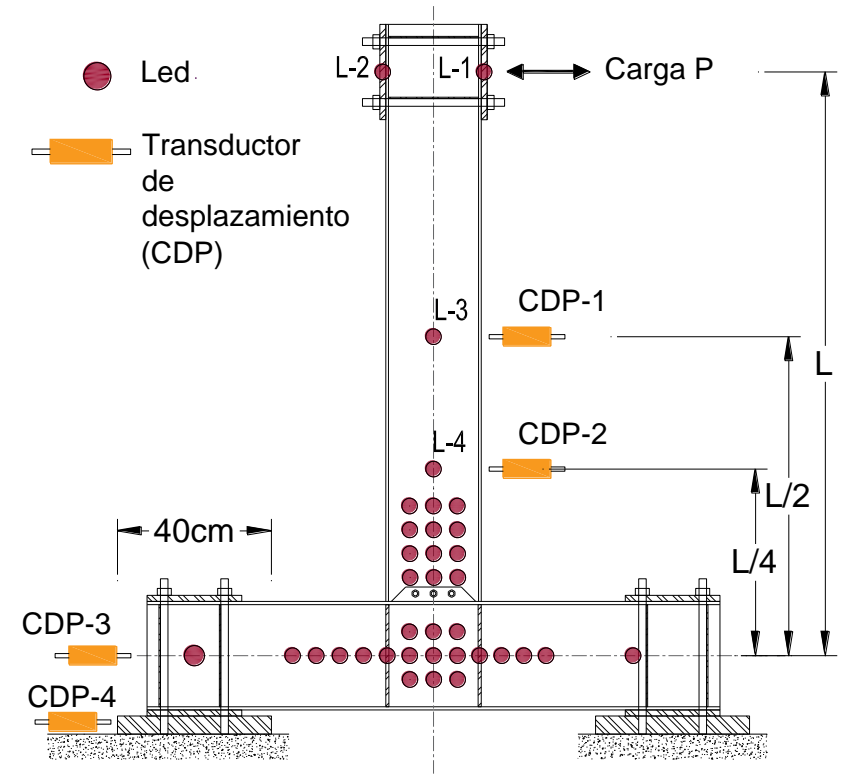

a) Espécimen CTP1

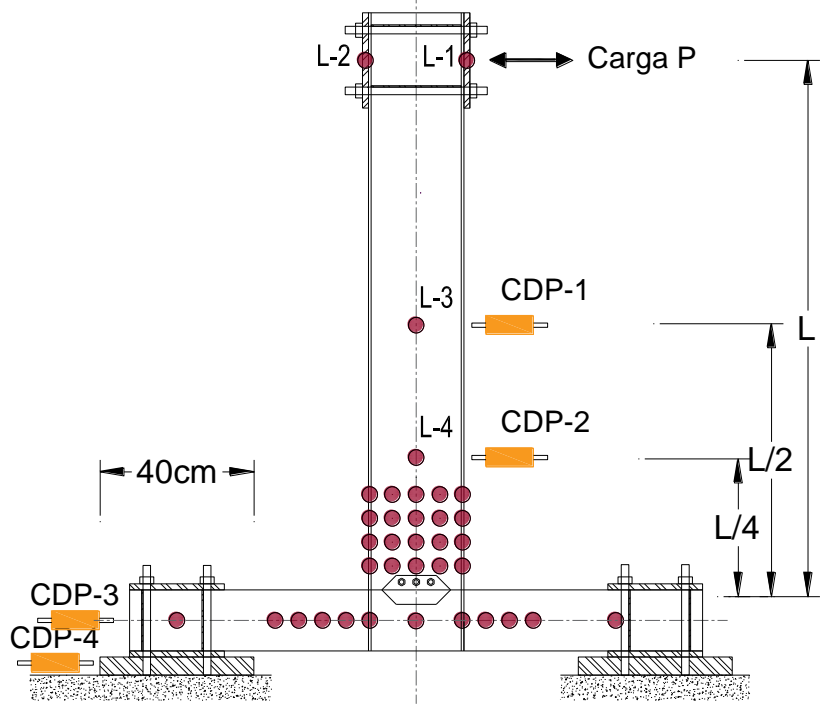

b) Espécimen CPA y CTA

Figura 4. Instrumentación empleada en los especímenes (cotas en centímetros)

\section{RESULTADOS EXPERIMENTALES}

En esta sección se discuten los resultados de las seis pruebas experimentales, agrupadas en función del tipo configuración. De manera que, aunque en cada caso se ensayaron dos especímenes, se discuten los resultados típicos y, posteriormente, se discutirán los pormenores analíticos. En la figura 5 se muestran las maniobras para la colocación de los especímenes desde un foso de almacenamiento hasta su posición en la losa de reacción. Por motivos de espacio, la descripción de la preparación sólo se incluye para este espécimen. Es posible consultar una discusión más detallada en Santiago (2020).

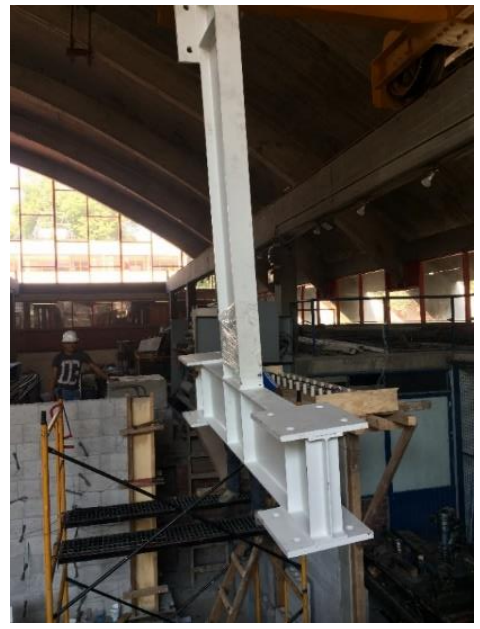

a) Traslado con grúa

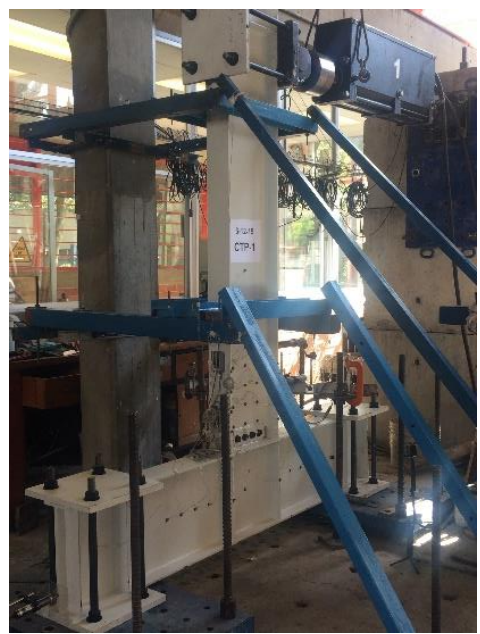

b) Colocación en la losa

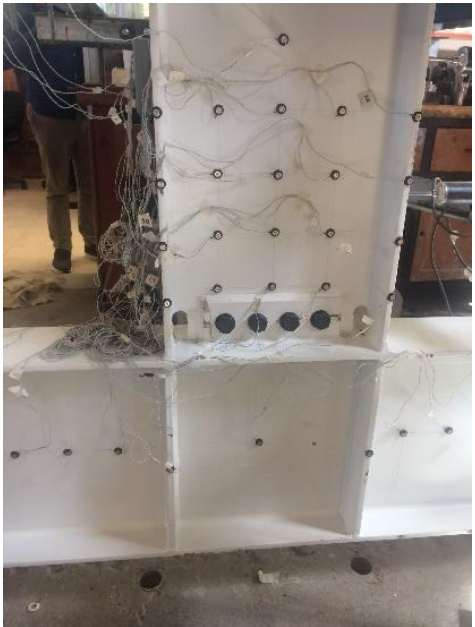

c) Instrumentación

Figura 5. Preparación de espécimen 


\section{Especímenes CTP1}

En la figura 6a se muestra la evolución de uno de los ensayes típicos de los especímenes CTP1-01, que se aproxima a la conexión precalificada WUF-W (AISC 358-16). El inicio de la fluencia se registró en el ciclo 1 del grupo 6 cuando se estaban aplicando 18.29 t; aunque en ese momento, el espécimen no mostraba daño físico. En el ciclo 1 del grupo 7 fue visible el daño en la conexión, que inició con deformaciones por cortante en la zona de panel cuando se estaba aplicando una carga de $21.65 \mathrm{t}$ (figura 6b). En el ciclo 1 del grupo 8 inició el pandeo local del patín de la trabe justo en la zona de la muesca en uno de los lados (figura 6c) y en el ciclo 2 del mismo grupo se identificó el pandeo en ambos patines.

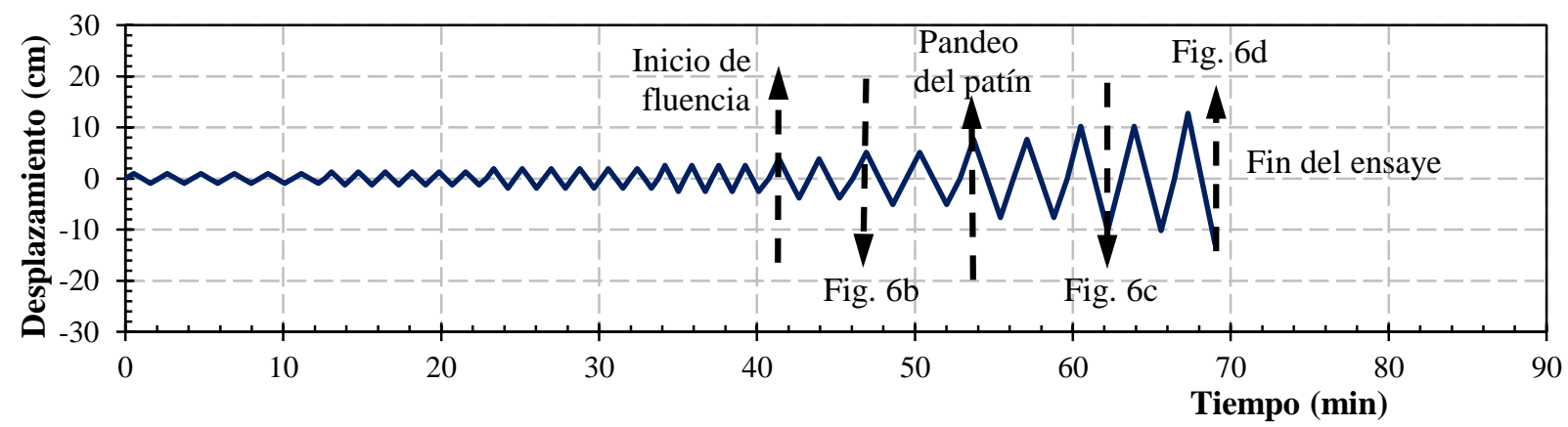

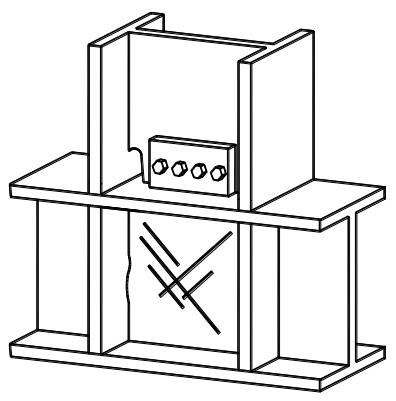

b) Daño en la zona de panel

a) Evolución de la prueba

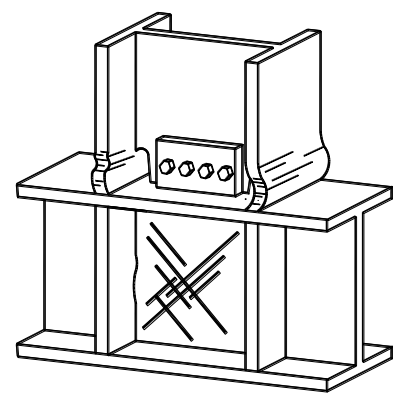

c) Pandeo del patín en la muesca

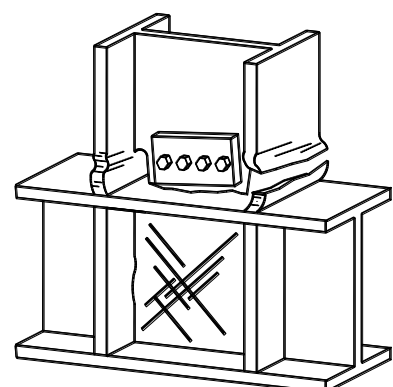

d) Fractura en el alma de la trabe

Figura 6. Reporte de daño típico del espécimen CTP1

Finalmente, cuando iniciaba el grupo 9 en el ciclo 1 se fracturó la soldadura que unía el alma de la trabe a los cartabones de la columna (figura 6d). El daño estuvo liderado por la fractura en el cordón de soldadura en el alma de la trabe que ocasionó en el mismo paso la fractura del patín de la trabe a $2 \mathrm{~cm}$ del cordón de la soldadura (figura 7). Con este daño se suspendió el ensaye sin que se formara una articulación plástica en la trabe.

En la figura 8 se muestran las curvas momento - rotación de ambos especímenes con la configuración CTP1. Se hace notar que, en ambos casos, el momento resistente superó el 0.8 del momento plástico $M_{p}$ y que la rotación que resistió fue mayor a 0.04 radianes. Por esto, pese a que la conexión desarrolló un mecanismo de falla indeseable, la conexión está relacionada con una respuesta que califica para ser usada en un marco dúctil conforme al manual AISC 341-16 (2016). 


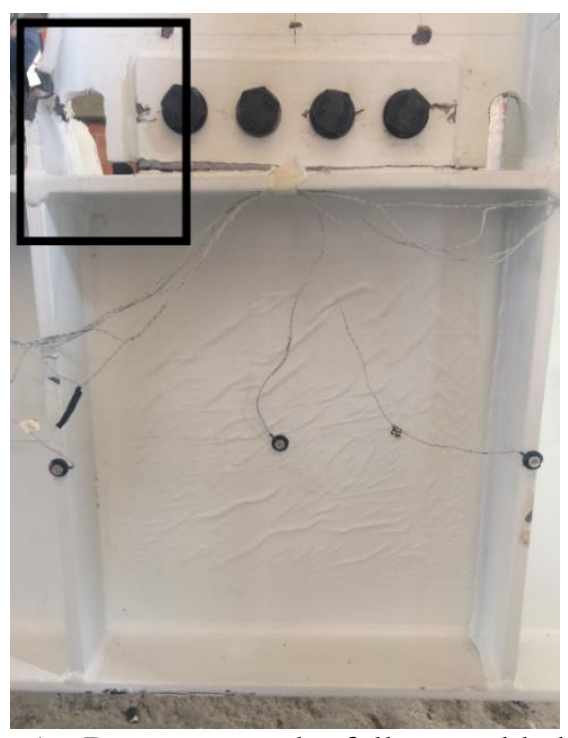

a) Daño en panel y falla en soldadura

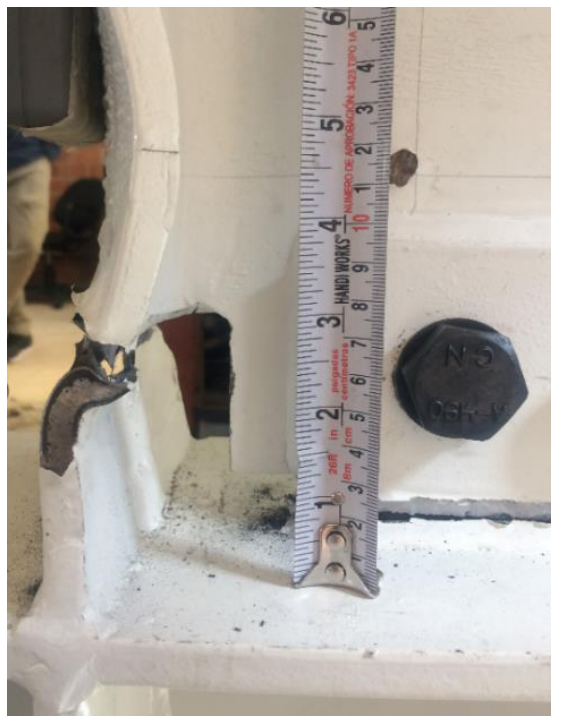

b) Acercamiento en la fractura del patín

Figura 7. Estado final típico de los especímenes CTP1

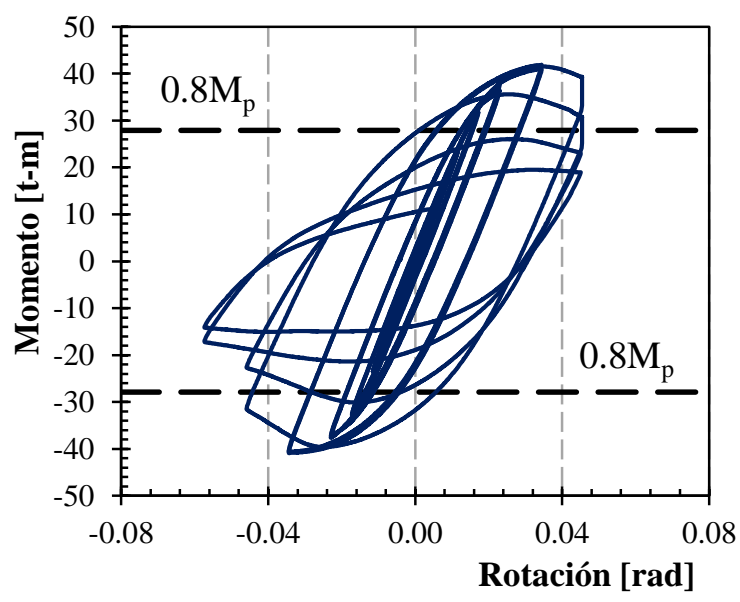

a) Espécimen 1

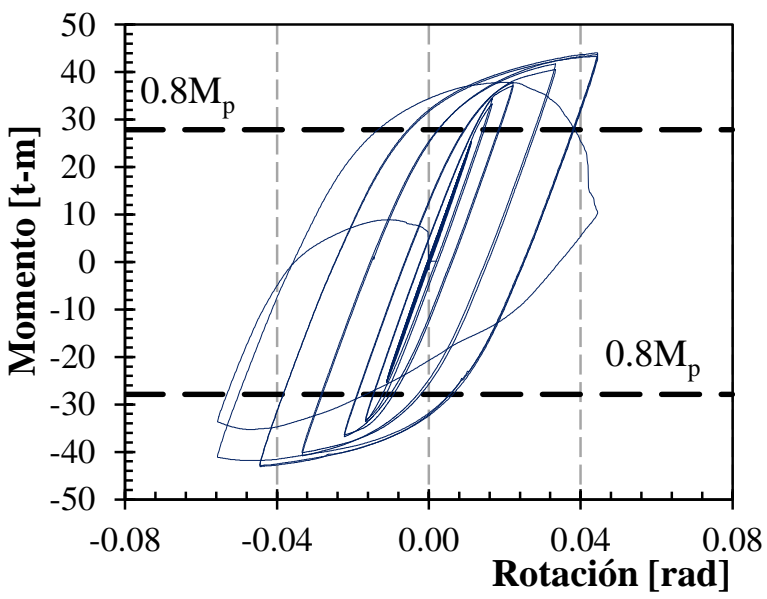

b) Espécimen 2

Figura 8. Curvas momento - rotación de la configuración CTP1

\section{Especímenes CTA}

Estos ensayes corresponden a un espécimen de conexión de la trabe al eje débil de una columna I. La conexión extrapola las recomendaciones de la conexión precalificada WUF-W del AISC 358-16 para unir la trabe con placas y cartabones. El inicio de la fluencia ocurrió en el primer ciclo del grupo 6, mientras se estaba aplicando una carga de 16.37 t. Físicamente, no se notaba daño en la trabe ni en la conexión, pese a que los aparatos reportaron una degradación de la rigidez.

El pandeo local en los patines de la trabe a la altura de la muesca ocurrió en forma visible en el segundo ciclo del grupo 7 con una deformación de $7.644 \mathrm{~cm}$ en el extremo libre de la trabe (Fig. 9b). El agrietamiento inicial en el alma en la muesca ocurrió en el último ciclo del grupo 8 (Fig. 9c). Finalmente, 
la fractura en el alma de la trabe en ambos extremos, a partir de la muesca ocurrió en el primer ciclo del grupo 9.

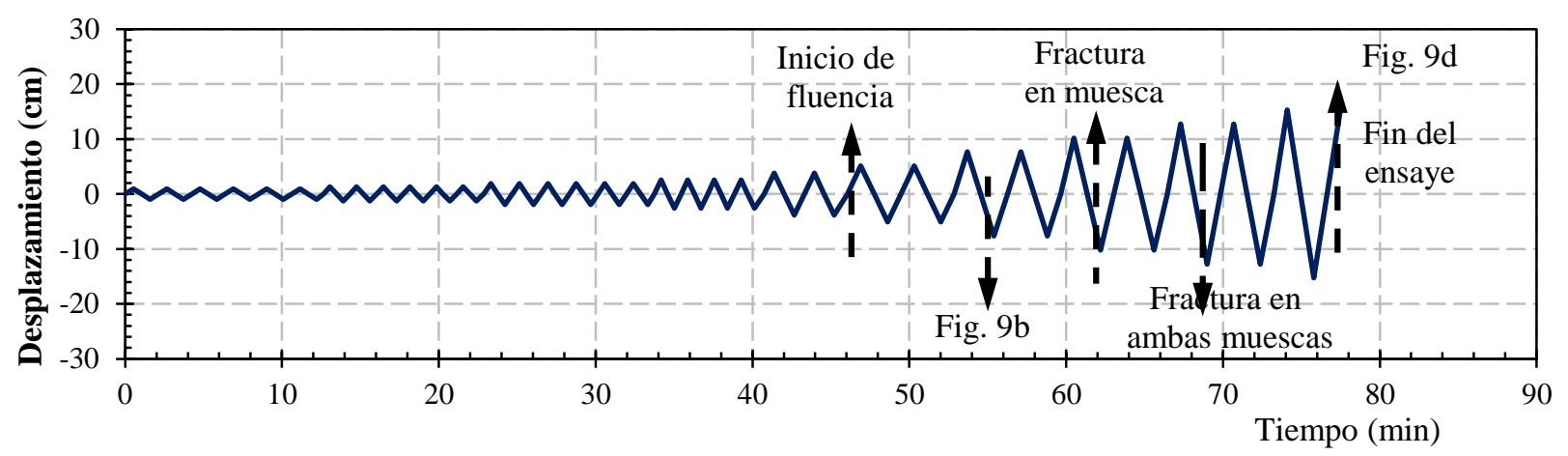

a) Evolución de la prueba

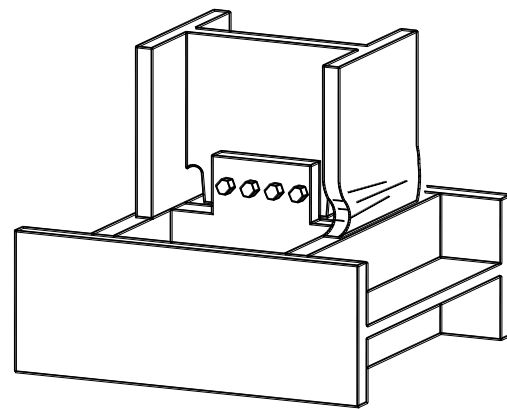

b) Pandeo de patín de trabe

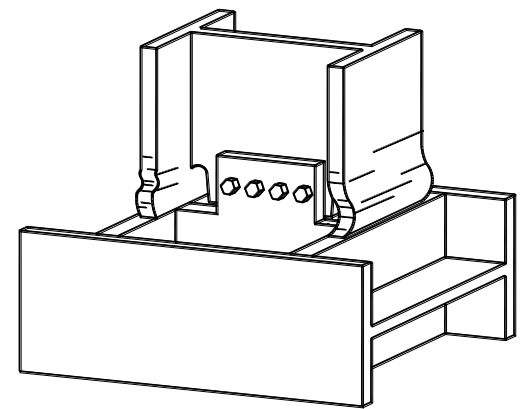

c) Daño en la zona de la muesca

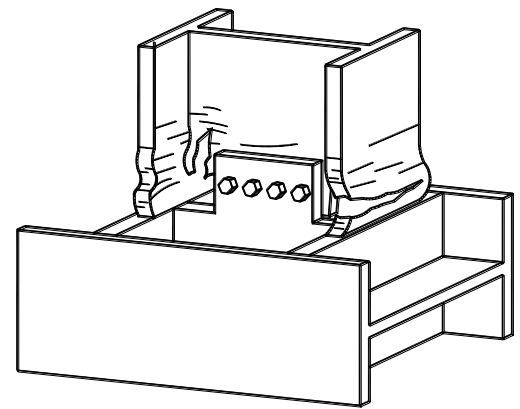

d) Fractura del patín de la trabe Figura 9. Reporte de daño típico del espécimen CTA

La falla de la conexión ocurrió en el primer ciclo del grupo 10, caracterizada por la fractura transversal del patín. La falla se situó a $2 \mathrm{~cm}$ de la soldadura corrida que une el patín con el cartabón transversal de la columna (Fig. 9d). En este ciclo se detuvo el ensaye debido a la pérdida de resistencia para salvaguardar el equipo (Fig. 10). Al final del ensaye, ambos patines reportaron pandeo en la zona de la muesca, donde se notaron fracturas de hasta $3 \mathrm{~cm}$ en el alma de la trabe. No se notó daño en la soldadura ni la formación de la articulación plástica en la trabe como se supone en la etapa de diseño de marcos dúctiles (e.g., Sec. 12.2.1, NTC-DCEA-2020).

En la figura 11 se muestran las curvas momento - rotación de ambos especímenes con la configuración CTA. El momento resistente superó el $0.8 M_{p}$ y la rotación que resistió fue superior a 0.04 radianes, por lo que podría ser considerada para usarse en marcos dúctiles conforme al manual AISC 34116 (2016), pese a la concentración de daño desarrollado. 


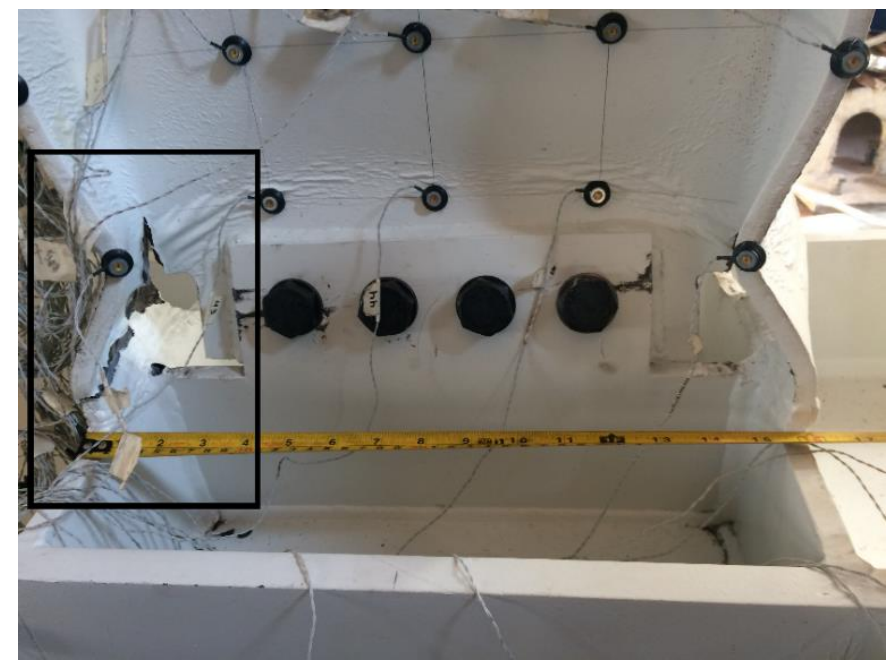

a) Daño en la zona de las muescas

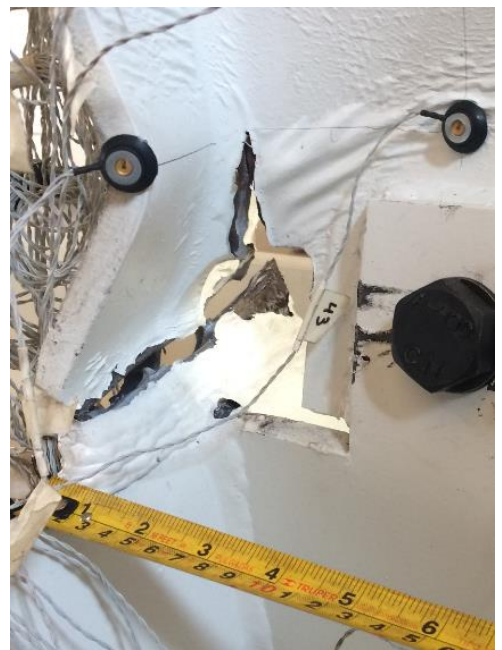

b) Acercamiento en la fractura del patín

Figura 10. Estado final típico de los especímenes CTA

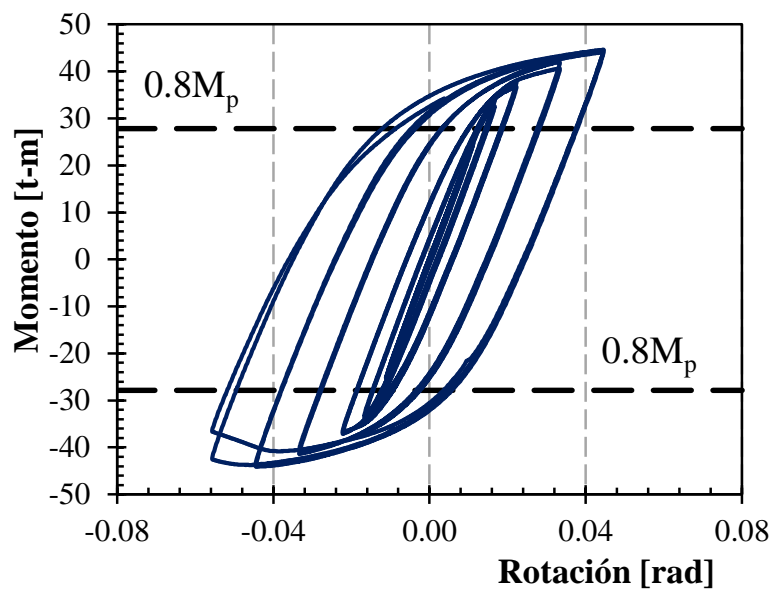

a) Espécimen 1

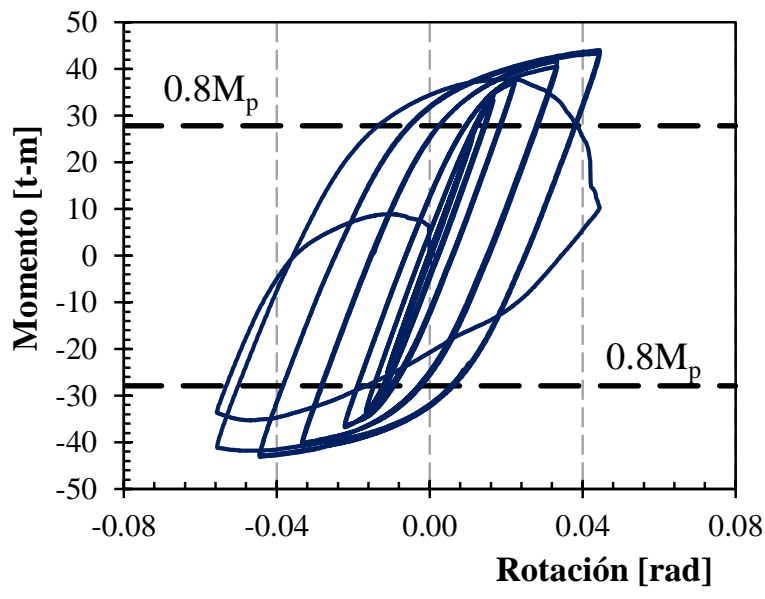

b) Espécimen 2

Figura 11. Curvas momento - rotación de la configuración CTA

\section{Especímenes CPA}

Esta configuración de conexión une la trabe al eje menor de la columna I mediante una placa trapezoidal. Los primeros cinco grupos de ciclos reportaron comportamiento elástico; de manera que la respuesta inelástica se registró en aparatos hasta el primer ciclo del grupo 6 cuando se aplicaba una carga de $16.732 \mathrm{t}$, pese a que físicamente el espécimen se notaba sin daño.

Como se muestra en la figura 12, la evolución del daño en la conexión inició con el pandeo del patín en la zona de la muesca en el primer ciclo del grupo 7 cuando el actuador aplicaba una carga de 18.66 toneladas (Fig. 12b). Físicamente, el pandeo de ambos patines se identificó hasta el primer ciclo del grupo 8 con una carga de 19.39 t (Fig. 12c). La fractura en ambas muescas ocurrió en el primer ciclo del grupo 9. 


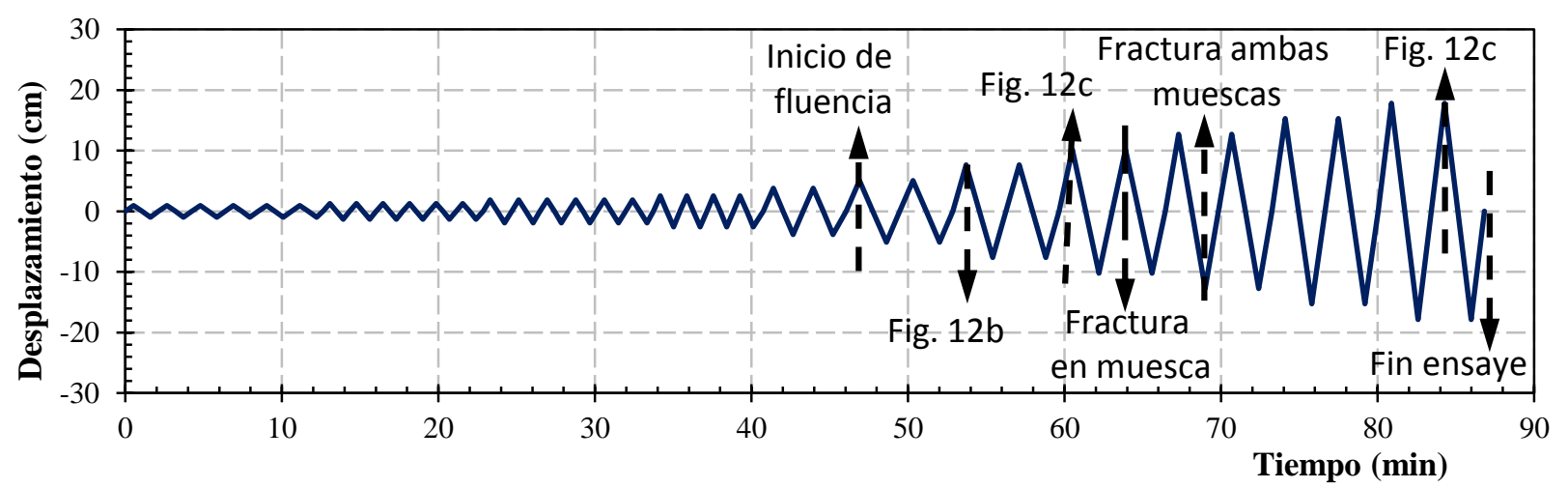

a) Evolución de la prueba

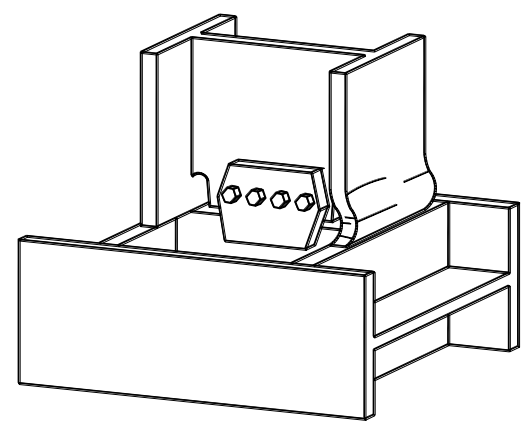

b) Pandeo de patín de trabe

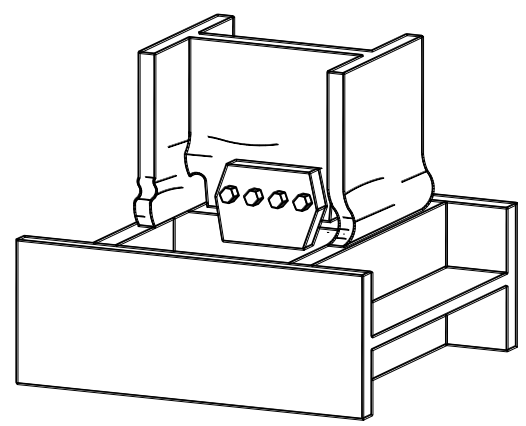

c) Daño en la zona de la muesca

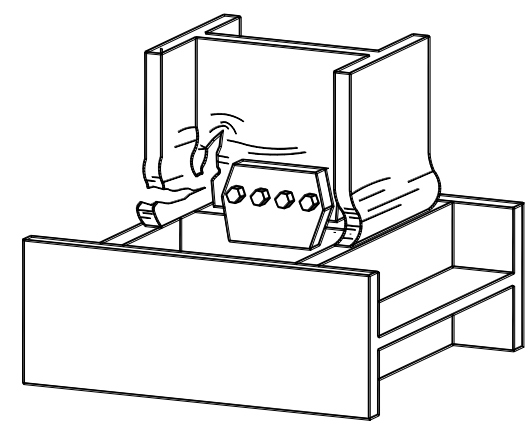

d) Fractura del patín de la trabe

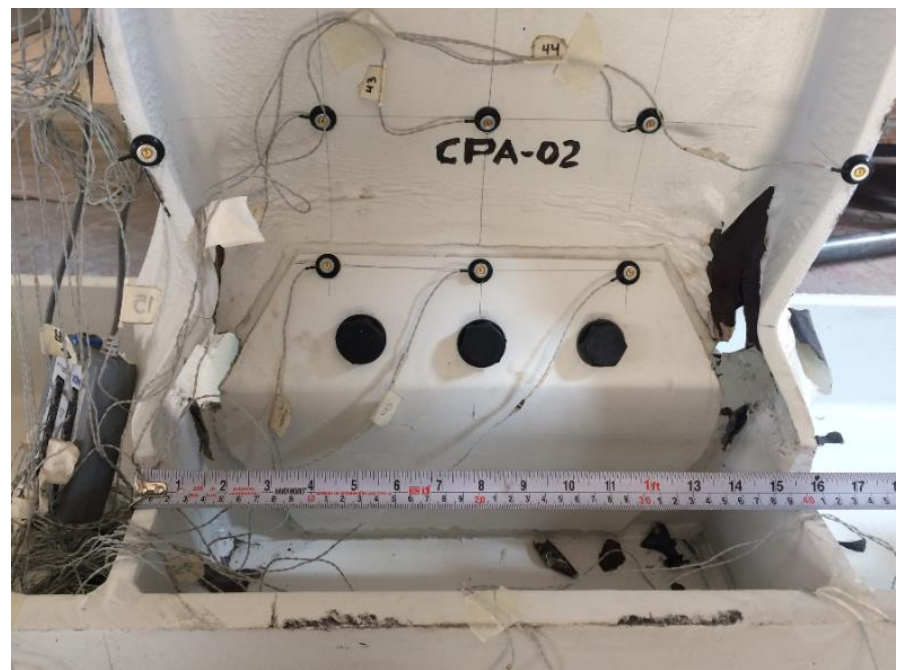

a) Daño en la zona de las muescas

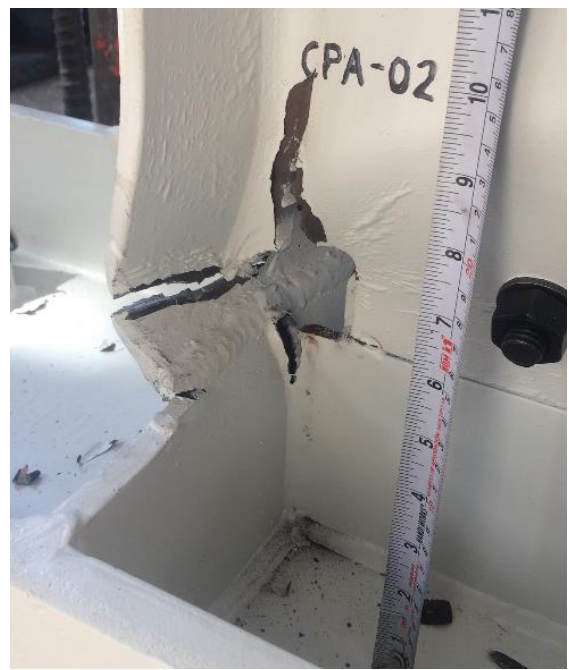

b) Fractura del patín

Figura 13. Estado final típico de los especímenes CPA

Durante el ensaye, se notó poco o nulo daño en el alma de la trabe en la zona donde se debía desarrollar la articulación plástica, sino que la evolución del mecanismo fue liderada por la fractura parcial en la zona de la muesca de uno de los patines de la trabe. El daño local ocurrió en el segundo ciclo del grupo 10 (Fig. 12d), por lo que fue posible completar los ciclos del grupo 10. 
En comparación con la configuración CTA, la conexión CPA soportó una mayor cantidad de ciclos, aunque la falla es significativamente similar (Fig. 13). De hecho, la configuración de la conexión CTA desarrolló una incursión inelástica mucho más estable como se nota en la curvas momento - rotación de los especímenes en la figura 14. El momento resistente también superó el $0.8 M_{p}$ y la rotación que resistió fue superior a 0.04 radianes, por lo que podría ser considerada para usarse en marcos dúctiles conforme al manual AISC 341-16 (2016).

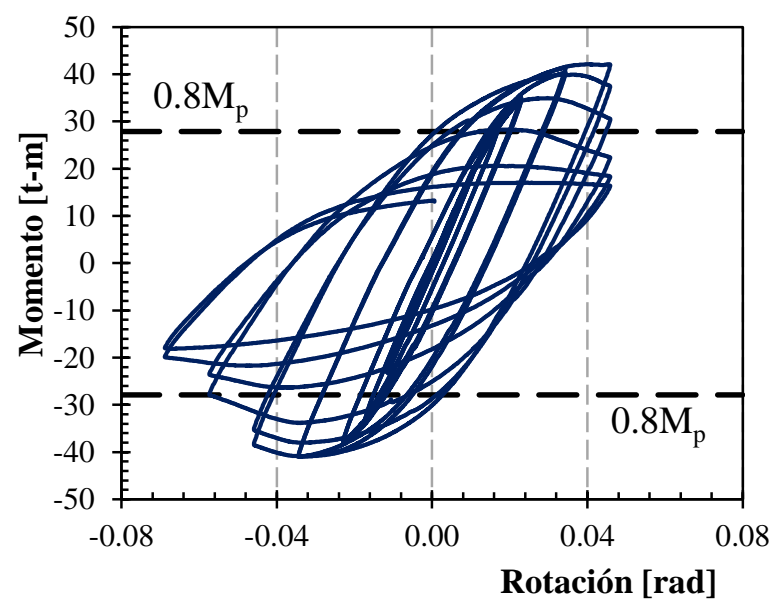

a) Espécimen 1

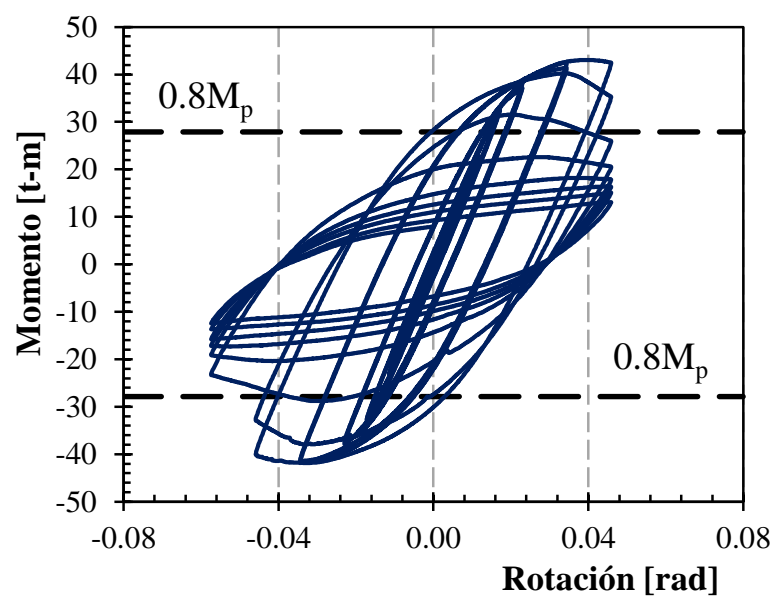

b) Espécimen 2

Figura 14. Curvas momento - rotación de la configuración CPA

\section{DISCUSIÓN DE RESULTADOS}

En la figura 15 se muestra la definición de los parámetros que se discuten en esta sección. Donde, $\varphi_{y}$ es la rotación asociada a la fluencia reportada en la prueba experimental; $\varphi_{\text {máx }}$ es la rotación máxima (positiva o negativa) asociada a medio ciclo de histéresis de la carga máxima; $K_{e}$ es la rigidez elástica (pendiente A-B); $K_{i n}$ es la rigidez inelástica (pendiente B-C) y $K_{\min }$ es la rigidez secante mínima inelástica (pendiente A-C) reportada en el último medio ciclo estable de la prueba experimental.

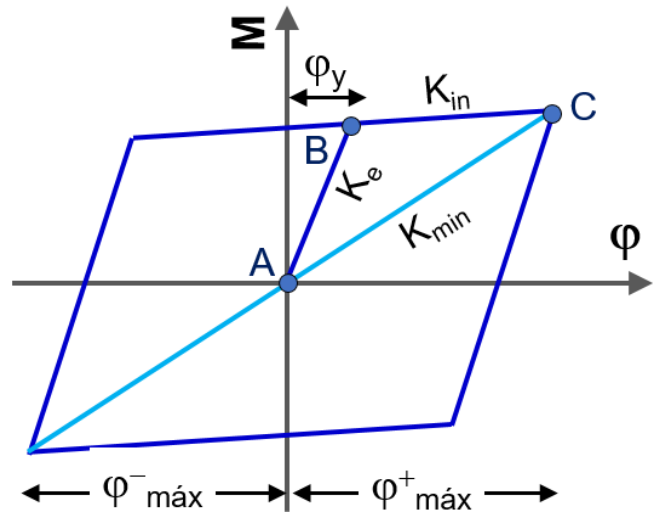

Figura 15. Definición de parámetros

Los parámetros discutidos se calcularon, a partir de los resultados experimentales, que se muestran en la tabla 2. La degradación de la rigidez elástica en el medio ciclo previo a la falla de las conexiones en 
todos los casos resultó ser cercano al 60 por ciento $\left(K_{\min } / K_{e} \approx 0.60\right)$. Esto se debe relacionar con la estabilidad de la incursión inelástica, pese a la inesperada concentración de daño en los patines. En cuanto a la pendiente inelástica, en modelos de articulaciones concentradas en vigas y columnas se permite modificar la pendiente B-C en un 3 por ciento de la pendiente elástica conforme a las NTC-DCEA-2020 (Apéndice F) para considerar los efectos del endurecimiento por deformación (consultar Tapia et al. 2016 para una discusión más amplia).

Con base en los resultados, la rigidez $K_{\text {in }}$ reportada en los experimentos podría ser incluso superior al 20 por ciento de la rigidez elástica $K_{e}$, lo que subraya la estabilidad de la incursión inelástica. Adicionalmente, conforme a la tabla 4.2.2 de las Normas Técnicas Complementarias para el Diseño por Sismo (NTC-DS-2020), los marcos dúctiles pueden ser diseñados para un factor de comportamiento sísmico de hasta $Q=4.0$. El mecanismo asociado a esa demanda de ductilidad está liderado por la formación de una articulación plástica en las trabes; de manera que, siguiendo un diseño por capacidad, las columnas y las conexiones deberían diseñarse con la máxima capacidad que puede desarrollar la trabe en ese mecanismo. Sin embargo, los resultados asocian la evolución de la falla con una concentración de esfuerzos en la conexión que limita las ductilidades a las que se muestran en la tabla 2. Esto es, pese a que en el proceso de diseño se siga estrictamente la filosofía del diseño por capacidad en el diseño de los marcos de acero (Sec. 12.2.1, NTC-DCEA-2020), la respuesta de las conexiones ensayadas limitaría la capacidad dúctil a magnitudes menores a $\varphi_{\max } / \varphi_{y}=\mu<4.0$.

Tabla 2. Respuesta inelástica de los especímenes ensayados

\begin{tabular}{|l|c|c|c|c|c|c|}
\hline \multicolumn{1}{|c|}{ Espécimen } & $K_{\min } / K_{e}$ & $\begin{array}{c}K_{\text {in }} \\
(\mathrm{t}-\mathrm{m} / \mathrm{rad})\end{array}$ & $K_{\text {in }} / K_{e}$ & $\begin{array}{c}\varphi_{y} \\
(\mathrm{rad})\end{array}$ & $\begin{array}{c}\left|\varphi_{\max }\right| \\
(\mathrm{rad})\end{array}$ & $\mu$ \\
\hline CTP1-01 & 0.4889 & 395.964 & 0.1949 & 0.0151 & 0.0502 & 3.26 \\
\hline CTP1-02 & 0.5010 & 428.348 & 0.2141 & 0.0179 & 0.0592 & 3.33 \\
\hline CTA-01 & 0.5421 & 447.122 & 0.2666 & 0.0170 & 0.0681 & 4.00 \\
\hline CTA-02 & 0.6387 & 526.601 & 0.2792 & 0.0171 & 0.0567 & 3.29 \\
\hline CPA-01 & 0.6259 & 496.269 & 0.2585 & 0.0169 & 0.0563 & 3.32 \\
\hline CPA-02 & 0.6447 & 557.648 & 0.2901 & 0.0178 & 0.0570 & 3.20 \\
\hline
\end{tabular}

En una conexión típica, la falla es controlada por los requisitos de detallado de la soldadura en la unión de los patines de la trabe a la columna, la soldadura de la unión del alma de la trabe a la columna y la forma y acabado de las muescas. Con base en los resultados, independientemente de que la conexión de la trabe se analizara al eje mayor o al eje menor de la columna, la concentración del daño estuvo liderada por los esfuerzos en el patín de la trabe a compresión.

En los reglamentos especializados (e.g. AISC 360-16; NTC-DCEA-2020), la fuerza máxima que puede resistir el patín por efectos del momento aplicado en la trabe se toma como $T_{p a t i n}=1.8 A_{f} F_{y}$; donde $A_{f}$ es el área transversal del patín y $F_{y}$ el esfuerzo de fluencia del material. En esa determinación se considera que:

i) El endurecimiento por deformación incrementará la resistencia nominal del momento plástico en un 30 por ciento.

ii) La placa que une el alma de la trabe es inefectiva en transferir el momento flexionante a la columna.

iii) El módulo de sección plástico de los patines $Z_{p}$ es aproximadamente el 70 por ciento del módulo de la sección plástico $Z$ de la sección completa. 
Entonces, si sólo los patines son efectivos para transferir el momento flexionante de la trabe a la columna (figura 16a), el máximo momento que podrían desarrollar los patines de la viga $M_{\text {patin }}$ se determina con la carga de los patines y el peralte de trabe $d$ como se discute en la ecuación 1 .

$T_{\text {patín }}=\frac{M_{\text {patinn }}}{d}=\frac{1.3\left(Z_{p} / 0.7\right) F_{y}}{d}=\frac{1.8 A_{p} d F_{y}}{d}=1.8 A_{p} F_{y}$

Esa evolución del daño se debe a que la conexión del alma de la trabe al patín (o cartabón) de la columna mediante una placa no es una transferencia directa de los esfuerzos por flexión soportados en el alma de la trabe. Los esfuerzos en el alma de la trabe se deben redireccionar hacia el patín para conseguir una transferencia hacia la columna (figura 16a), puesto que el alma de la trabe no es efectiva para transferir los esfuerzos. De hecho, la placa de conexión vista en corte obligaría a un cambio de eje de los esfuerzos como se muestra en la figura $16 \mathrm{~b}$.

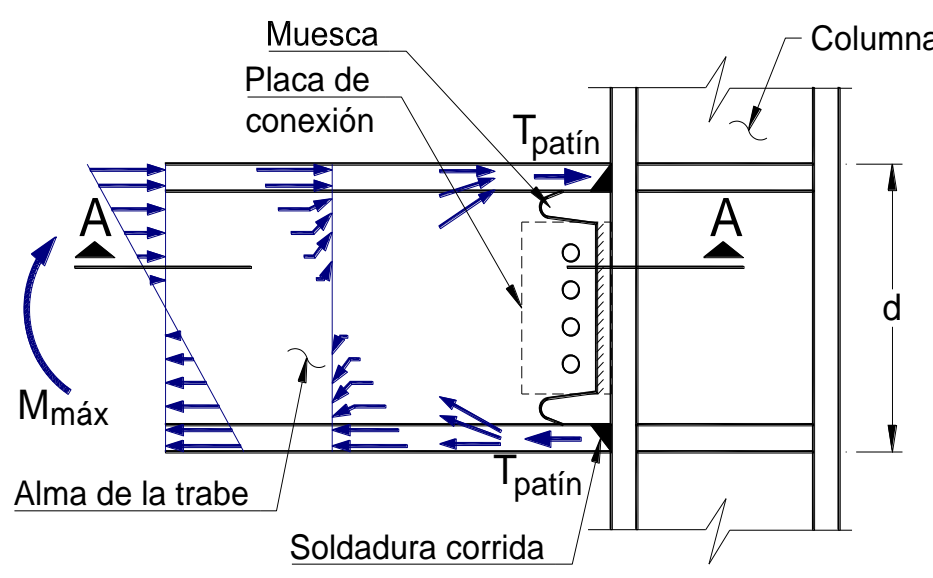

a) Concentración de esfuerzos

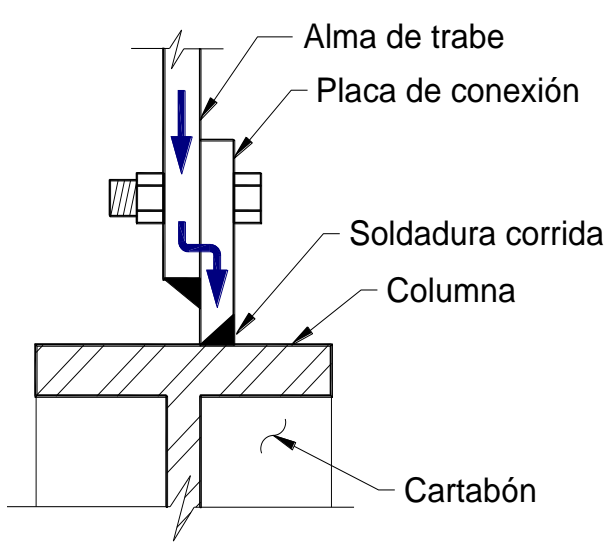

b) Corte $\mathrm{A}-\mathrm{A}$

Figura 16. Distribución de esfuerzos típica en la conexión

Por lo anterior, la capacidad a momento de las conexiones ensayadas es sólo una porción del momento plástico esperado $M_{p e}$ (ecuación 2). Esto justifica el hecho de que la falla en los experimentos ocurra sin el desarrollo de una articulación plástica en la trabe, puesto que el momento plástico esperado $M_{p e}$ que ocasiona la articulación plástica en la trabe es mayor que el momento que logra desarrollar el patín plástico esperado $M_{\text {patín }}=T_{\text {patind }}$ (figura 16a).

$\frac{T_{p e}}{T_{\text {patin }}}=\frac{M_{\text {pe }} / d}{M_{\text {patin }} / d}=\frac{1.3 Z F_{y}}{1.3\left(Z_{p} / 0.7\right) F_{y}}=\frac{Z}{Z_{p} / 0.7}$

Se ha comprobado experimentalmente (Popov y Tsai 1989; Engelhardt y Husain 1993), que cuando la relación $Z_{p} / Z$ es menor a 0.70 las conexiones desarrollan una respuesta frágil con una capacidad de rotación incluso menor a 0.015 radianes. Las proporciones de los especímenes ensayados aquí fueron iguales a $Z_{p} / Z=0.54$ en la conexión CTP (a eje mayor de la columna) y son iguales a $Z_{p} / Z=0.65$ en las conexiones CTA y CPA (a eje menor de la columna).

Entonces, para evitar la evolución de la falla es necesario incrementar la aportación de los patines al módulo de sección plástico hasta proporciones $Z_{p} / Z>0.70$, agregando cartabones perpendiculares a los patines y/o cubreplacas. Adicionalmente, con el propósito de privilegiar el desarrollo de la articulación en la trabe, los requisitos del suplemento AWS D1.8 (2016) y el manual AISC 358 (2016) establecen 
condiciones geométricas que se complementan entre sí para la colocación de soldadura en conexiones como las ensayadas en esta investigación. Los requisitos geométricos pretenden evitar una concentración de daño ocasionado por la muesca en la zona de los patines.

En la figura 17a se muestran los requisitos de ambas publicaciones en un acercamiento de la conexión del patín inferior y se complementan con la descripción de variables de la tabla 3. En la figura 17b se muestra un acercamiento típico de la misma zona de las configuraciones previas a los ensayes para contrastar las diferencias.

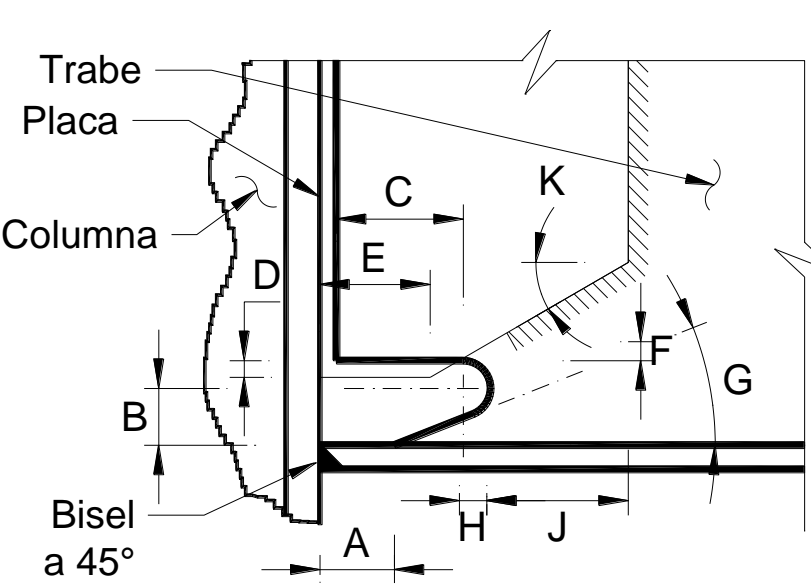

a) Recomendaciones geométricas

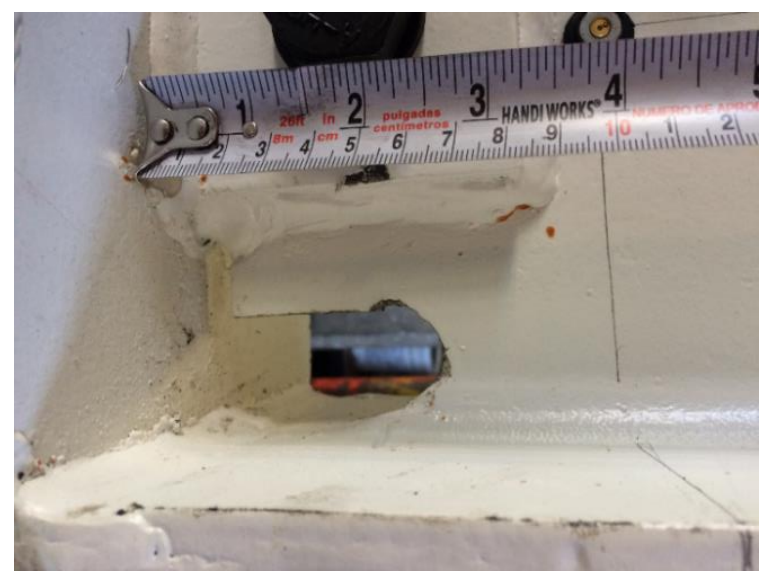

b) Detalle típico previo al ensaye

Figura 17. Imperfecciones en la muesca

En los orificios de acceso a la soldadura como muescas o ranuras es recomendable que se minimicen las imperfecciones ocasionadas por los cortes o perforaciones, vigilando que las dimensiones, tolerancias y los perfiles cumplan los requisitos de la tabla 3. Esas imperfecciones propiciaron la concentración del daño que evolucionó en el pandeo y fractura de los patines reportados en esta investigación y, por consiguiente, la falla prematura de la conexión.

Tabla 3. Recomendaciones del AWS D1.8 (2016) y AISC 358 (2016) para la colocación de soldadura

\begin{tabular}{|c|c|}
\hline Dimensión & Recomendación \\
\hline $\mathrm{A}$ & $\begin{array}{l}\text { La distancia al paño del patín debe ser la mayor distancia entre } 0.5 t_{p} \text { más el } \\
\text { espesor del patín } t_{p} \text { ó } 0.5 t_{p} \text { más } 12 \mathrm{~mm}(1 / 2 ")\end{array}$ \\
\hline B & $\begin{array}{l}\text { La dimensión mínima debe ser la mayor entre } 0.75 t_{p} \text { ó } 20 \mathrm{~mm}(3 / 4 \text { ") y la } \\
\text { dimensión máxima debe ser } t_{p}+6 \mathrm{~mm}(1 / 4 \text { ") }\end{array}$ \\
\hline $\mathrm{C}$ & La longitud de la muesca debe ser $3 t_{p} \pm 12 \mathrm{~mm}(1 / 2 ")$ \\
\hline $\mathrm{D}$ & $\begin{array}{l}\text { El traslape de la placa y la muesca debe ser al menos } 6 \mathrm{~mm}(1 / 4 \text { ") y máximo } \\
12 \mathrm{~mm}(1 / 2 ")\end{array}$ \\
\hline E & Tamaño de la placa $25 \mathrm{~mm}(1 ”)$ mínimo \\
\hline $\mathrm{F}$ & $\begin{array}{l}\text { La distancia mínima del paño a la muesca al cordón de soldadura de filete } \\
\text { es } 12 \mathrm{~mm}(1 / 2 ") \text { y la máxima es } 25 \mathrm{~mm}(1 \text { ") }\end{array}$ \\
\hline $\mathrm{G}$ & El ángulo de la muesca y el patín no debe exceder de $25^{\circ}$ \\
\hline $\mathrm{H}$ & El radio mínimo de la muesca debe ser $10 \mathrm{~mm}\left(3 / 8^{\prime \prime}\right)$ \\
\hline $\mathrm{K}$ & El ángulo de la placa de conexión debe ser $30^{\circ} \pm 10^{\circ}$ \\
\hline $\mathrm{J}$ & $\begin{array}{l}\text { La distancia mínima del paño de la muesca al final de la placa de conexión } \\
\text { es } 50 \mathrm{~mm}(2 \text { ") }\end{array}$ \\
\hline
\end{tabular}




\section{CONCLUSIONES}

En este artículo se discuten los resultados de pruebas experimentales a conexiones de acero trabe columna en escala natural de algunas de las configuraciones más utilizadas en México. El estudio pretende evaluar el comportamiento de conexiones existentes de columna I a trabe I basadas en las configuraciones típicas para identificar posibles procedimientos que mejoren la respuesta. Las configuraciones ensayadas se establecieron mediante una encuesta con algunos de los despachos de cálculo y fabricantes de estructuras de acero más reconocidos en el país. En el consenso, se establecieron tres configuraciones: un espécimen con una conexión a momento conectando al eje mayor de la columna y dada la incertidumbre local, se consideraron dos conexiones a momento al eje menor de la columna. En cada caso se ensayaron dos especímenes. Las principales contribuciones de esta investigación son las siguientes:

- La evolución de los ensayes experimentales de los especímenes reporta una concentración de daño en la zona de la conexión, sin que se desarrolle una articulación plástica en la trabe. Más específicamente, la falla es liderada por el pandeo de los patines en la zona de la muesca, lo que evolucionó en: i) fisuras en el alma de la trabe desde las imperfecciones de la muesca, ii) la fractura del área total del patín y, en algunos casos, iii) en fisuras en los cordones de soldadura. Esto representa una respuesta inelástica inesperada, debido a que la capacidad del sistema fue menor a la que se establece si se sigue un diseño por capacidad.

- Pese a la concentración de daño, la incursión inelástica desarrollada estuvo relacionada con ciclos estables con degradaciones de rigidez y resistencia graduales y dúctiles. Sin embargo, la ductilidad reportada en los experimentos resultó limitada; por lo que la aplicación de estas configuraciones de conexiones a marcos rígidos debería estar restringida a diseños con un factor de comportamiento sísmico de hasta $Q=3.0$.

- Dada la configuración de las conexiones, la placa que une el alma de la trabe resultó inefectiva en transferir el momento flexionante a la columna, por lo que la capacidad de la conexión dependió altamente de la capacidad de los patines de la trabe. Se hizo notar la conveniencia de que las proporciones entre el módulo de sección plástico $Z$ y la aportación de los patines al módulo de sección plástico $Z_{p}$ sean mayores a $Z_{p} / Z>0.70$. El incremento necesario de la aportación de los patines al módulo de sección plástico $\left(Z_{p}\right)$ se recomienda mediante la adición de cartabones o placas a los patines de las trabes en la zona de la muesca.

- Finalmente, se hizo notar la importancia de seguir recomendaciones especializadas en el detallado de la conexión para mejorar la respuesta durante el proceso de fabricación. En particular, se listaron condiciones geométricas que pretenden evitan el pandeo de los patines de la trabe en la zona de la muesca. En particular, las imperfecciones ocasionadas por los cortes o perforaciones de las conexiones analizadas parecen haber favorecido la concentración del daño.

\section{AGRADECIMIENTOS}

Los autores desean agradecer al Ing. Pablo Gómez Urquiza, Ing. Luis Gatica, Ing. Humberto Arevalo, M.I. Carlos Moss Vélez, Ing. Gabriel A. Guerra Vanegas, M.I. Javier Alonso García, M.C. Carlos Alfredo Tapia García, M.I. Raúl Jean Perrilliat, Dr. Rodolfo Valles Mattox, M.I. Jesus Miguel Yacaman Mendez, Ing. Raúl Granados Granados e Ing. Fernando González Roser por su apoyo, asesorías y opiniones durante las etapas de esta investigación. 


\section{REFERENCIAS}

AISC-341 (2016), Seismic Provisions for Structural Steel Buildings, ANSI/AISC 341-16. American Institute of Steel Construction. Chicago, IL, USA.

AISC-358 (2016), Prequalified Connections for Special and Intermediate Steel Moment Frames for Seismic Applications, ANSI/AISC 358-16. American Institute of Steel Construction. Chicago, IL, USA.

AISC-360 (2016), Specification for Structural Steel Buildings, ANSI/AISC 360-16. American Institute of Steel Construction. Chicago, IL, USA.

AWS D8.1 (2009), Structural Welding Code - Seismic Supplement. An American National Standard. AWS D1.8/D1.8 M. American Welding Society D1 Committee on Structural Welding. $2^{\text {nd }}$ edition.

Bertero V.V.; Anderson J.C. y Krawinkler H. (1994), "Performance of Steel Building Structures during the Northridge Earthquake Report No. UCB/EERC-94/09. Berkeley: Earthquake Engineering Research Center, University of California.

Engelhardt M.D. y Husain A.S. (1993), "Cyclic Loading Performance of Welded Flange-Bolted Web Connections", Journal of Structural Engineering, American Society of Civil Engineers, Vol. 119. No. 12, pp. 3537-3550. https://doi.org/10.1061/(ASCE)0733-9445(1993)119:12(3537)

FEMA-350 (2000), Recommended Seismic Design Criteria for New Steel Moment-Frame Buildings, FEMA-350, Federal Emergency Management Agency, Washington, DC.

FEMA-355C (2000), State of the Art Report on Systems Performance of Steel Moment Frames Subject to Earthquake Ground Shaking, FEMA-355C, prepared by the SAC Joint Venture for the Federal Emergency Management Agency, Washington, DC.

FEMA-355D (2000), State of the Art Report on Connection Performance, FEMA-355D, prepared by the SAC Joint Venture for the Federal Emergency Management Agency, Washington, DC.

FEMA-355F (2000), State of the Art Report on Performance Prediction and Evaluations of Steel Moment Frame Buildings, FEMA-355F, prepared by the SAC Joint Venture for the Federal Emergency Management Agency, Washington, DC.

García J.S. y Tapia E. (2019), "Respuesta inelástica de marcos dúctiles con contraviento concéntrico", Revista de Ingeniería Sísmica, SMIS. No. 100. Pp. 51-70. https://doi.org/10.18867/ris.100.478

Martín del Campo T., (2013), Estudio experimental de conexiones de acero a momento de vigas I con columna rectangular HSS, Tesis de maestría, Universidad Autónoma Metropolitana - Azcapotzalco.

NTC-DCEA-2020 (2020), Normas Técnicas Complementarias para el Diseño y Construcción de Estructuras de Acero con Comentarios, Gaceta Oficial de la Ciudad de México, Vigésima primera época, No. 381,7 de julio.

NTC-DS-2020 (2020), Normas Técnicas Complementarias para el Diseño por Sismo con Comentarios, Gaceta Oficial de la Ciudad de México, Vigésima primera época, No. 361, 9 de junio.

Osteraas J. S.M. y Krawinkler H. (1989), "The Mexico Earthquake of September 19, 1985 - Behavior of Steel Buildings", Earthquake Spectra, Vol. 5, No. 1. Pp. 51 - 88. https://doi.org/10.1193/1.1585511

Popov E.P. y Tsai K.C. (1989) "Performance of Large Seismic Steel Moment Connections Under Cyclic Loads", Engineering Journal, $2^{\text {nd }}$ Quarter, American Institute of Steel Construction.

SAC (1995), "Interim Guideless: Evaluation, Repair, Modification and Design of Welded Steel Moment Frame Structures. Program to Reduce the Earthquake Hazards of Steel Moment Frame Structures. Federal Emergency Management Agency. Report FEMA 267/SAC-95-02. SAC Joint Venture. Sacramento, California.

SAC (1997), "Interim Guideless Advisory No. 1: Supplement to FEMA 267. Program to Reduce the Earthquake Hazards of Steel Moment Frame Structures. Federal Emergency Management Agency. Report FEMA 267A/SAC-96-03. SAC Joint Venture. Sacramento, California.

Santiago A. (2020), "Comportamiento de Conexiones de Acero Conforme a la Práctica Mexicana", Tesis de Maestría, Universidad Nacional Autónoma de México. En proceso.

Tapia E. y Tena A. (2001), "Comparación de los efectos observados durante los sismos de México (1985), Northridge (1994) y Kobe (1995) y su impacto en las Normas de Diseño para Estructuras Metálicas 
del RCDF-2001”, Memorias, XIII Congreso Nacional de Ingeniería Sísmica, ID. IV-08. Guadalajara, Jal. Noviembre

Tapia E., García J.S., y Del Rincón A. (2016), "Estudio paramétrico del modelado inelástico de contravientos", Revista de Ingeniería Sísmica, SMIS. No. 94. Pp. 49-74. doi: dx.doi.org/10.18867/ris$\underline{0}$

Tapia E. y García J.S. (2019), "Comportamiento de estructuras de acero durante los sismos de septiembre del 2017”, Revista de Ingeniería Sísmica, No. 101, pp. 36-52. https://doi.org/10.18867/ris.101.499

Tremblay R., Filiatrault A., Timler P. y Bruneau M., (1995), "Performance of steel structures during the 1994 Northridge earthquake", Canadian Journal of Civil Engineering, Vol. 22, pp. 338-360. https://doi.org/10.1139/195-046 